\title{
Alternativas de manejo expectante de la rotura prematura de membranas antes de la viabilidad en embarazos únicos
}

\author{
Adriana Doren V. ${ }^{1}$, Jorge Carvajal C. ${ }^{2}, P h D$. \\ 1 Programa de Obstetricia y Ginecología, ${ }^{2}$ Unidad de Medicina Materno Fetal, División de Obstetricia y Ginecología, \\ Facultad de Medicina, Pontificia Universidad Católica de Chile.
}

\section{RESUMEN}

La rotura prematura de membranas (RPM) antes de la viabilidad fetal constituye una complicación obstétrica de baja incidencia, pero que se asocia a una alta morbimortalidad materna y perinatal. No existen actualmente guías estandarizadas para el manejo expectante de esta patología, y las prácticas reportadas en la literatura para el enfrentamiento de este escenario clínico son variables. Aquí presentamos una revisión de la literatura de los últimos 10 años y proponemos una pauta de manejo para la RPM antes de la viabilidad basada en la evidencia disponible.

\section{PALABRAS CLAVE: Rotura prematura de membranas, segundo trimestre del embarazo, parto prematuro, infección perinatal}

\section{SUMMARY}

Preterm premature rupture of membranes (PPROM) before fetal viability is a low incidence complication of pregnancy, but associated with a high maternal and fetal morbidity and mortality. Currently standard guidelines for expectant management of this pathology are not available, thus the reported clinical management of this clinical situation are not uniform. We present a ten years literature review and we propose a guideline for management of PPROM before fetal viability based on current evidence.

\section{KEY WORDS: Premature rupture of membranes, second trimester of pregnancy, preterm delivery, perinatal infection}

\section{INTRODUCCIÓN}

La rotura prematura de membranas (RPM) se define como la solución de continuidad de la membrana corioamniótica antes del inicio del trabajo de parto. La RPM de pretérmino corresponde a aquella que ocurre antes de las 37 semanas de gestación. La RPM previabilidad se refiere a la que acontece antes del límite de viabilidad neonatal establecida, que en nuestro hospital correspondería a las 24 semanas de gestación.

Si bien la incidencia de RPM antes de las 24 semanas es baja $(0,37 \%)(1)$, su asociación a complicaciones maternas y fetales significativas, la hacen una patología de relevancia clínica y ética $(1,2)$. Gracias a los avances en medicina neonatal, el pronóstico de los recién nacidos post RPM previabilidad ha mejorado, y el manejo expectante de 
esta patología ha cobrado mayor interés. De hecho, distintos grupos de medicina perinatal han publicado en los últimos años sus resultados maternos y fetales en este escenario clínico.

El manejo de las pacientes con RPM antes de la viabilidad no se ha establecido en base a sólida evidencia científica, generando gran heterogeneidad de las intervenciones utilizadas como parte del manejo expectante; incluso, en muchas partes del mundo se plantea la interrupción del embarazo ante el diagnóstico de RPM previabilidad, debido al pronóstico reservado para el feto/recién nacido. De este modo, definir un consenso acerca del enfrentamiento de los embarazos con RPM antes de la viabilidad, se hace difícil.

El objetivo de esta publicación es revisar la literatura de la última década, para evaluar los distintos manejos definidos como expectantes en la RPM antes de la viabilidad, y asociar dichos manejos con los resultados maternos y fetales. En base a los hallazgos propondremos un algoritmo de manejo para esta situación clínica.

\section{MÉTODOS}

Obtención de la información: Se efectuó la búsqueda en PubMed usando los siguientes términos MeSh: "fetal membranes, premature rupture" AND "pregnancy trimester, second". Se limitó la búsqueda a estudios en humanos, en inglés o español, y se restringió a estudios publicados entre el año 2000 y 2011. Se agregaron referencias secundarias que cumplían con los criterios de inclusión expuestos. Selección de estudios: Se incluyó todos los estudios retrospectivos o prospectivos que evaluaran los resultados maternos y/o fetales de la RPM en el segundo trimestre y describieran el manejo expectante utilizado en estas pacientes. Se excluyó aquellos estudios referidos sólo a RPM iatrogénica, los reportes de casos clínicos con bajo número de casos o aquellos que sólo se refirieran a RPM de gemelos, a factores de riesgo de hipoplasia pulmonar o que utilizaran intervenciones alternativas específicas como amniocentesis, esponjas cervicales o parches biológicos. Si bien se excluyó estudios que hicieran alusión sólo a embarazos múltiples, se incluyó trabajos que tuvieran dentro de su muestra embarazos múltiples, siempre y cuando la mayoría de la población en estudio fuese de embarazos únicos.

\section{RESULTADOS}

Resultado de la búsqueda bibliográfica. Detecta- mos un total de 130 estudios, de los cuales 30 parecían apropiados al revisar el resumen. La lectura del texto completo mostró que sólo 10 cumplían con los criterios de inclusión propuestos. Dos de los 10 estudios seleccionados corresponden a la misma muestra pero con distintos resultados a analizar. Además, incluimos 3 referencias secundarias que cumplían con los criterios propuestos.

Seleccionamos 4 estudios que reportan técnicas alternativas para el escenario clínico en cuestión, para comentar dichas estrategias en la discusión, destacando que 2 de estos estudios incluyen en su muestra tanto RPM iatrogénica como espontánea. Nuestra búsqueda no obtuvo ningún estudio randomizado.

Características de los estudios. De los 13 estudios seleccionados que se refieren al manejo expectante de la RPM del segundo trimestre, 2 se definen como prospectivos en su metodología $(3,4)$, el resto son todos estudios retrospectivos de fichas clínicas o bases de datos (5-15). Dos publicaciones corresponden a la misma población estudiada, una analiza los resultados neonatales con un seguimiento a dos años (5) y el otro tiene como objetivo evaluar los factores pronósticos de los nacidos vivos de la muestra en cuestión (6).

Como se puede ver en la Tabla I, existen variaciones en los criterios de inclusión y exclusión, porque se incluyen distintos rangos de edad gestacional a la que ocurrió RPM, aunque todas dentro del segundo trimestre, previabilidad. Algunos estudios no definen el límite inferior de edad gestacional de RPM que usaron como criterio de inclusión (4,7-10). Dos estudios no definen los criterios de exclusión usados $(7,10)$, y tres no establecen si incluyen o no embarazos múltiples (8-10). Un estudio incluye en su muestra pacientes con RPM producida posterior a procedimientos invasivos (15\%) (7). Un trabajo definió como criterio de exclusión una latencia menor a 14 días (8), pero en sus resultados también describe los resultados tomando en cuenta la población con latencia larga y corta. Cuatro estudios incluyen embarazos múltiples $(4-6,11)$. De los trabajos que especifican sus criterios de exclusión, los más frecuentemente usados son RPM pos amniocentesis (AMCT), trabajo de parto al ingreso o latencia menor a 24 horas, corioamnionitis clínica al ingreso, incompetencia cervical o cerclaje, metrorragia importante, solicitud de terminar el embarazo y anomalías fetales. Una sola publicación es multicéntrica, pues se realizó en dos hospitales sudafricanos (4), y dos no especifican donde se llevaron a cabo (3-9). 
Tabla I

CARACTERÍSTICAS CLÍNICAS DE LOS ESTUDIOS ANALIZADOS

\begin{tabular}{|c|c|c|c|c|c|c|}
\hline Cita & $\begin{array}{l}\text { Período } \\
\text { de estudio }\end{array}$ & $\begin{array}{l}\text { Pacientes } \\
\text { (fetos) }\end{array}$ & Gemelar & $\begin{array}{l}\text { EG RPM } \\
\text { (semanas) }\end{array}$ & $\begin{array}{c}\text { Manejo } \\
\text { hospitalizado }\end{array}$ & $\begin{array}{c}\text { Tocolisis } \\
\text { (\%) }\end{array}$ \\
\hline 3 & 1995-1998 & 163 & No & $15-28$ & ¿? & $\begin{array}{c}\mathrm{Si} \leq 32 \text { sem } \\
\quad \% \text { ¿? }\end{array}$ \\
\hline 4 & ¿? & $\begin{array}{c}78 \\
(81)\end{array}$ & $\mathrm{Si}$ & $<28$ & $\mathrm{Si}$ & No \\
\hline $5-6$ & 1998-2005 & $\begin{array}{l}63 \\
(71)\end{array}$ & $\mathrm{Si}$ & $14-24+6$ & $\mathrm{Si}$ & $\begin{array}{c}\mathrm{Si} \\
100 \%<24 \text { sem }\end{array}$ \\
\hline 7 & $1996-2002$ & $\begin{array}{l}43 \\
(57)\end{array}$ & $\mathrm{Si}$ & $<24$ & $\begin{array}{c}\text { No, ingreso } \\
\text { inicial y a las } 24 \\
\text { semanas }\end{array}$ & No \\
\hline 8 & $200-2005$ & 98 & ¿? & $<24$ & ¿? & ¿? \\
\hline 9 & $1994-2004$ & 36 & No & $<24$ & $\mathrm{Si}$ & $\begin{array}{c}\mathrm{Si} \\
\% \text { ¿? }\end{array}$ \\
\hline 10 & $1992-1997$ & 28 & ¿? & $<25$ & $\mathrm{Si}$ & ¿? \\
\hline 11 & 1995-1999 & 57 & $\mathrm{Si}$ & $14-24$ & $\mathrm{Si}$ & $\begin{array}{c}\mathrm{Si} \\
7 \%\end{array}$ \\
\hline 12 & $1995-2001$ & 25 & No & $16-24$ & $\mathrm{Si}$ & $\begin{array}{c}\mathrm{Si} \\
12 \%\end{array}$ \\
\hline 13 & 1998-2003 & $\begin{array}{c}49 \\
(29)^{*}\end{array}$ & No & $18-24+6$ & $\mathrm{Si}$ & $\begin{array}{c}\mathrm{Si} \\
14,3 \%\end{array}$ \\
\hline 14 & 1997-1999 & 66 & No & $18-23$ & $\mathrm{Si}$ & ¿? \\
\hline 15 & 1991-1996 & 59 & No & $14-23$ & ¿? & $\begin{array}{c}\mathrm{Si} \geq 22 \text { sem } \\
11 \%\end{array}$ \\
\hline
\end{tabular}

¿?: El estudio no describe con precisión esta información. EG: Edad gestacional. RPM: Rotura prematura de membranas. *: Manejo expectante.

Dos trabajos de cohorte retrospectiva seleccionaron como grupo de estudio a nacidos vivos de madres con RPM del segundo trimestre con el objetivo de identificar factores pronósticos de sobrevida neonatal $(6,10)$.

Manejo antenatal. Para el diagnóstico y evaluación inicial, en todos los estudios se hizo el diagnóstico de RPM a través de la visualización de salida de líquido amniótico por el orificio cervical externo, con o sin confirmación con algún test. En 8 de las publicaciones revisadas se tomó cultivos vaginales al ingreso (3-5,7-9,11-13), describiéndose en dos de ellos la repetición periódica de dicho examen $(8,12)$. Sólo cuatro estudios establecen la toma de parámetros inflamatorios maternos al ingreso (2, $5,12,15)$, con repetición periódica en dos $(5,15)$. En 6 protocolos se realizaron ecografías periódicas para evaluar el líquido amniótico y el crecimiento fetal, generalmente semanal $(3-5,8,10,12)$.

Manejo hospitalizado versus ambulatorio: Como se observa en la Tabla I, en todas las publicaciones analizadas las embarazadas se mantuvieron hospitalizadas durante todo el período de latencia, excepto en un estudio realizado en EEUU en el cual, luego de una evaluación hospitalizada inicial para descartar corioamnionitis, las pacientes fueron dadas de alta con indicación de visitas frecuentes, reingresadas a las 24 semanas, para permanecer luego hospitalizadas hasta el final del embarazo (7). Antibióticos profilácticos: En todos los estudios se comenta el uso de antibióticos profilácticos antenatales, y en 10 de las 12 poblaciones evaluadas la gran mayoría de las pacientes los recibió (promedio: $76,8 \%$, rango: $12,3-100 \%$ ), pero con esquemas muy 
distintos entre sí (Tabla II). Por ejemplo, 2 poblaciones recibieron antibióticos hasta el parto $(4,12)$, una de ellas ampicilina más eritromicina (12) y la otra amoxicilina más metronidazol (4). Otros tres estudios indicaron antibióticos por 7 días al ingreso con esquemas variados $(5,6,7,13,14)$, y en el caso de un trabajo norteamericano, éstos se indicaban a las 24 semanas si la paciente no los había recibido previamente (7). Dos autores describen el uso de antimicrobianos al ingreso ajustado o suspendido según el resultado del cultivo vaginal $(3,9)$. Un trabajo describe la indicación de antibióticos al ingreso pero no el tiempo de administración (8) y otros 3 manifiestan su uso sin mayor detalle $(10,11,15)$.

Tocolisis y corticoides: En la Tabla I se puede observar que en 7 estudios se administró tocolisis, reportándose su uso en 11 a $100 \%$ de las pacientes (promedio: 28,9\%). En la mayoría de los que se implementó esta terapia fue por dinámica uterina previa a una edad gestacional establecida. Sólo en un trabajo su uso fue universal acompañando la administración de corticoides (5).

Se describe el uso de corticoides para madurez pulmonar alcanzada la viabilidad fetal en todos los estudios (Tabla I), excepto en uno en que no se hace mención de su uso (9). La utilidad de administración antenatal de corticoides a pacientes en riesgo de parto prematuro incluyendo RPM, está ampliamente avalada en la literatura $(16,17)$, por lo que no analizaremos este tema mayormente.

Estudios complementarios: Sólo un trabajo plantea la realización de AMCT para evaluar madurez pulmonar y descartar infección intraamniótica (IIA) a todas las pacientes que alcanzaran las 32 semanas de gestación (3). Otro sólo describe que su utilización no es de rutina (10).

Resultados maternos. En general pocos estudios describen en detalle la morbilidad materna asociada a RPM previabilidad. Dos publicaciones hacen alusión al inicio de trabajo de parto prematuro espontáneo, ocurriendo en un 50,9 y $73 \%$ de los casos respectivamente $(5,11)$.

La incidencia de corioamnionitis clínica es el resultado materno más reportado, describiéndose una incidencia de entre un $21-64 \%(4,7,11,14,15)$. Un estudio demostró que la corioamnionitis clínica

Tabla II

USO DE ANTIBIÓTICOS PROFILÁCTICOS

\begin{tabular}{|c|c|c|c|c|}
\hline Cita & $\begin{array}{l}\text { \% que recibió } \\
\text { antibióticos }\end{array}$ & Antibióticos & $\begin{array}{c}\text { Vía de } \\
\text { administración }\end{array}$ & Tiempo \\
\hline 3 & $100 \%$ & Ampicilina o Clindamicina & ¿? & $\begin{array}{l}7 \text { días o hasta } \\
\text { cultivo SGB (-) }\end{array}$ \\
\hline 4 & $100 \%$ & Amoxicilina + Metronidazol & $¿ ?$ & Hasta el parto \\
\hline $5-6$ & $93,6 \%$ & $\begin{array}{l}\text { Penicilina o Amoxicilina o } \\
\text { Cefalosporinas }\end{array}$ & ¿? & 7 días \\
\hline 7 & $\begin{array}{l}63 \% \text { al ingreso } \\
23 \% \text { a las } 24 \text { sem }\end{array}$ & $\begin{array}{l}\text { Ampicilina/Sulbactán, luego, } \\
\text { Amoxicilina/Clavulánico } \\
\text { (Clindamicina si hay alergia) }\end{array}$ & EV, luego VO & $\begin{array}{c}48 \text { h EV, luego } 5 \\
\text { días VO }\end{array}$ \\
\hline 8 & $100 \%$ & Eritromicina & ¿? & ¿? \\
\hline 9 & $100 \%$ & Ampicilina/Sulbactán, luego según cultivo & ¿? & $i ?$ \\
\hline 10 & $89 \%$ & ¿? & ¿? & $i ?$ \\
\hline 11 & $12,3 \%$ & ¿? & $¿ ?$ & $i ?$ \\
\hline 12 & $100 \%$ & Ampicilina + Eritromicina & 7 dias EV, luego VO & Hasta el parto \\
\hline 13 & $70,5 \%$ & Amoxicilina o Eritromicina & EV luego VO & $\begin{array}{l}48 \text { h EV, luego } 5 \\
\text { días VO }\end{array}$ \\
\hline 14 & $46,9 \%$ & $\begin{array}{l}\text { Ampicilina/Amoxicilina } \pm \text { Eritromicina } \\
\text { (Clindamicina si hay alergia) }\end{array}$ & EV, luego VO & $\begin{array}{l}48 \text { h EV, luego } 5 \\
\text { días VO }\end{array}$ \\
\hline 15 & $23 \%$ & ND & ¿? & ¿? \\
\hline
\end{tabular}

¿? El estudio no describe con precisión esta información. 
no aumenta a mayor período de latencia, y que el $46 \%$ se relaciona a la presencia de cultivo vaginales positivos (4). Sí existiría asociación entre corioamnionitis clínica e histológica en un $89 \%$ de los casos (4). Por otro lado, un trabajo austriaco demostró que el $68,4 \%$ de las placentas con corioamnionitis histológica se relaciona a cultivos vaginales positivos (5). Un estudio francés no encontró diferencias en el índice de líquido amniótico (ILA) entre las pacientes con y sin corioamnionitis, explicando esto por el pequeño tamaño muestral (13), pues esto contradice lo usualmente descrito en la literatura (18). Se reportó un shock séptico materno en contexto de corioamnionits (13).

La tasa de fiebre postparto varía entre un 17,5\% (11) y un $32 \%$ (12). La tasa de endometritis descrita es de $17 \%$ (4); indicamos como referencia que esta tasa es significativamente mayor que la incidencia de endometritis reportada luego de partos de término sin RPM (1,6\%) (19).

En cuanto al desprendimiento prematuro de placenta normo inserta (DPPNI) se describe una ocurrencia de 4,5-10,2\% (14,13). La incidencia de retención de placenta varía entre 9,1 (14) y 10,5\% (11), con un $7,6 \%$ de hemorragia posparto (14). En nuestro grupo de estudios no se reportan muertes maternas.

Resultados perinatales Sobrevida perinatal y factores pronósticos. La sobrevida perinatal observada en los estudios incluidos varía entre $2-54 \%$, con sobrevida de los recién nacidos (RN) al alta entre 2-47\% (Tabla III). Si se evalúa el resultado perinatal por grupos divididos según la edad gestacional (EG) a la que ocurre la RPM (Tabla IV), la sobrevida perinatal y de los $\mathrm{RN}$ al alta es mayor en el grupo con una RPM ocurrida entre las 20 a 24 semanas versus el grupo en que la RPM ocurre bajo las 20 semanas, y la mortalidad fetal es menor a mayor EG de RPM. La mortalidad neonatal no parece relacionarse con la EG a la que ocurre la RPM; sin embargo, estudios que comparan el grupo de niños sobrevivientes versus no sobrevivientes pos RPM del segundo trimestre muestran resultados contradictorios en cuanto a la relación entre EG media de RPM y resultado perinatal, con algunos reportando mayor EG de RPM en el grupo sobreviviente $(p<0,05)(4,10)$, otros a mayor EG de RPM en el grupo que no sobrevivió $(p<0,001)$ (11), y algunos sin diferencias significativas entre ambos grupos (7).

En un estudio realizado en el Hospital de Miami en que los pacientes fueron divididas en 2 grupos según EG de RPM (18-21 y 22-23 semanas), se reportó una diferencia significativa entre ambos grupos en peso de nacimiento, nacidos vivos (NV), sobrevida perinatal y niños vivos al alta en beneficio del grupo con mayor EG de RPM ( $p<0,001)$ (14).

Un estudio sudafricano prospectivo de cohorte que incluyó pacientes con RPM hasta las 28 semanas, confirmó que la sobrevida perinatal es mayor a mayor EG de RPM, con una sobrevida de $76 \%$ si ocurría a $\geq$ de 26 semanas versus $35 \%$ si ésta sucedía entre las 20 y 26 semanas $(p=0,0003)$ (4). Cabe destacar que un $48 \%$ de las muertes neonatales ocurren durante las primeras 24 horas de vida (4).

Otros estudios buscaron establecer factores pronósticos dividiendo la población estudiada según la sobrevida perinatal. Cinco de los trabajos que dividieron su población en "sobrevivientes" y "no sobrevivientes" observaron que una mayor EG al parto y mayor peso de nacimiento son factores que mejoran el pronóstico de sobrevida perinatal $(4,5,7,12,13)$. Tres estudios demostraron que la ausencia de oligohidramnios (OHA) mejora la sobrevida perinatal $(10,12,13)$ y tres que una mayor latencia al parto se asocia a mejor pronóstico $(4,7,13)$. Sin embargo, un estudio francés observó que en los casos con latencia menor a 20 días la mortalidad era menor que con una latencia mayor a 20 días, sin diferencia significativa entre grupos (10). Sólo dos de estos estudios determinaron que el APGAR al nacer $(5,13)$ y que el número de cursos de corticoides, eran estadísticamente mayores en el grupo sobreviviente (13).

En Australia decidieron analizar el grupo de RPM bajo las 24 semanas que lograra una latencia mayor a 14 días, lo que correspondía a un $51 \%$ de las pacientes con este diagnóstico. En este subgrupo la tasa de nacidos vivos es de $80 \%$ y un $70 \%$ de sobrevida al alta. En esta población seleccionada no se observan diferencias en la presencia de OHA, EG de RPM, peso de nacimiento, EG al parto, presencia de corioamnionitis histológica, uso de corticoides antenatales ni en latencia al parto entre el grupo que sobrevive al alta y el que no (8).

Otros estudios demostraron que la sobrevida al alta disminuye significativamente con corioamnionitis histológica $(5,6,9)$, reportándose esta alteración hasta en el $75 \%$ de las placentas estudiadas $(9,15)$, y que su presencia se asocia a una PCR materna elevada de ingreso (15).

Los reportes son contradictorios en cuanto a la relación entre la EG de RPM y la latencia al parto, mostrándose relaciones directamente proporcionales $(p=0,022)(14)$ otras inversas $(p=0,0003)(4), y$ otras sin diferencias significativas (11). Un estudio describe que la leucocitosis y PCR elevada maternas se asocian a menor latencia al parto (15).

Antibióticos profilácticos y resultados perinatales. Dos estudios retrospectivos austriacos en una misma población de RPM del segundo trimestre repor- 
Tabla III

RESULTADO PERINATAL

\begin{tabular}{|c|c|c|c|c|c|c|}
\hline CITA & $\begin{array}{c}\text { EG RPM } \\
\text { (semanas) }\end{array}$ & $\begin{array}{l}\text { EG parto } \\
\text { (semanas) }\end{array}$ & $\begin{array}{c}\text { Latencia días } \\
\text { Mediana } \\
\text { (rango) }\end{array}$ & $\begin{array}{c}\text { Nacidos vivos } \\
(\%)\end{array}$ & $\begin{array}{l}\text { Sobrevida } \\
\text { perinatal } \\
(\%)\end{array}$ & $\begin{array}{c}\text { Sobrevida } \\
\text { al alta } \\
(\%)\end{array}$ \\
\hline 3 & ¿? & ¿? & ¿? & ¿? & 54 & ¿? \\
\hline 4 & 23,3 & 26,7 & $\begin{array}{c}12,5 \\
(1,5-154)\end{array}$ & 74,1 & 43 & ¿? \\
\hline $5-6$ & 21,2 & 23,1 & $\begin{array}{c}4 \\
(0-118)\end{array}$ & 35 & ¿? & 17 \\
\hline 7 & 22 & 25,8 & $\begin{array}{c}13 \\
(0-96)\end{array}$ & 61,4 & ¿? & 47 \\
\hline 8 & 19,8 & 28,4 & $\begin{array}{c}55 \\
(33-77)\end{array}$ & 62 & ¿? & 28,5 \\
\hline 9 & 19 & ¿? & $\begin{array}{c}19,7 \\
(0-189)\end{array}$ & 19,4 & ¿? & 13,9 \\
\hline 10 & 21,6 & 27,1 & 39,4 & ¿? & ¿? & $\begin{array}{l}64 \mathrm{de} \\
\text { los NV }\end{array}$ \\
\hline 11 & 20,3 & ¿? & $\begin{array}{c}6 \\
(1-161)\end{array}$ & 47,4 & ¿? & 26,3 \\
\hline 12 & 22,7 & ¿? & ¿? & ¿? & 32 & ¿? \\
\hline 13 & 21,1 & 23,2 & $\begin{array}{c}14 \\
(0-119)\end{array}$ & ¿? & ¿? & 24 \\
\hline 14 & ¿? & ¿? & $\begin{array}{c}62 \\
(12-646)\end{array}$ & 30,3 & 19,7 & 18,2 \\
\hline 15 & 21 & ¿? & $\begin{array}{c}1 \\
(0-90)\end{array}$ & ¿? & 2 & 2 \\
\hline $\begin{array}{l}\text { Promedio } \\
\text { (Rango) }\end{array}$ & $\begin{array}{c}21,2 \\
(19-13,3)\end{array}$ & $\begin{array}{c}25,7 \\
(23,1-28,4)\end{array}$ & $\begin{array}{c}22,7 \\
(1-62)\end{array}$ & $\begin{array}{c}47,1 \\
(19,4-74,1)\end{array}$ & $\begin{array}{c}30,1 \\
(2-54)\end{array}$ & $\begin{array}{l}26,8 \\
(2-64)\end{array}$ \\
\hline
\end{tabular}

EG: Edad gestacional. RPM: Rotura prematura de membranas. ¿? El estudio no describe con precisión esta información. NV: Nacidos vivos.

taron una mayor latencia media al parto en el grupo de pacientes que recibió antibióticos, con algunas diferencias según el antibiótico recibido: latencia media de 14 días con penicilina (49\%); 16 días con amoxi-clavulánico (32\%); 7 días con cefalosporinas (13\%) y 1 día sin antibióticos (6\%). Cabe destacar que en este estudio no se identificó ningún caso de enterocolitis necrotizante entre los RN $(5,6)$. No se observó una diferencia significativa en el uso de antibióticos antenatales entre el grupo de RN que sobrevivieron y el que no (5). Otro estudio concluye que los antibióticos prenatales es uno de los factores más importantes para mejorar la sobrevida perinatal $(p=0,05)$, sin describir que esquema antimicrobiano fue utilizado (10).

En el estudio realizado en Miami se encontró un tendencia a prolongar la latencia con el uso de antibióticos no significativa, pero no se demostró un efecto protector en la sobrevida perinatal y morbilidad neonatal (14). En dos trabajos se observó una tendencia a mayor cobertura con antibióticos antenatales en el grupo de niños que sobrevivieron al alta sin ser esto significativo $(6,12)$.

Morbilidad neonatal y secuelas a largo plazo. La incidencia de alteraciones pulmonares que requirieran intubación y ventilación mecánica fue altísima entre los recién nacidos vivos, llegando a reportarse un $78 \%$ (10), con una incidencia de síndrome de distrés respiratorio (SDR) que fluctúa entre $42-83 \%$ $(7,10,12,13)$. El $70 \%$ de las muertes neonatales fue por SDR e hipoplasia pulmonar (10). La prevalencia de hipoplasia pulmonar reportada varía entre un 


\section{Tabla IV}

RESULTADOS PERINATALES SEGÚN EDAD GESTACIONAL A LA QUE OCURRIÓ LA ROTURA PREMATURA DE MEMBRANAS

\begin{tabular}{|c|c|c|c|c|c|c|c|c|c|c|c|c|}
\hline \multirow[b]{2}{*}{ Cita } & \multicolumn{3}{|c|}{$>24$ semanas } & \multicolumn{3}{|c|}{ 20-24 semanas } & \multicolumn{3}{|c|}{$<20-21$ semanas } & \multicolumn{3}{|c|}{ Global } \\
\hline & MF & MNN & SP & MF & MNN & SP & MF & MNN & SP & MF & MNN & SP \\
\hline 4 & $\begin{array}{l}50 \% \\
(7 / 14)\end{array}$ & $\begin{array}{r}28,6 \% \\
(4 / 14)\end{array}$ & $\begin{array}{c}21,4 \% \\
(3 / 14)\end{array}$ & $\begin{array}{c}36,4 \% \\
(8 / 22)\end{array}$ & $\begin{array}{c}27,3 \% \\
(6 / 22)\end{array}$ & $\begin{array}{c}36,4 \% \\
(8 / 22)\end{array}$ & $\begin{array}{c}13,3 \% \\
(6 / 45)\end{array}$ & $\begin{array}{l}33,3 \% \\
(15 / 45)\end{array}$ & $\begin{array}{l}53,3 \% \\
(24 / 45)\end{array}$ & $\begin{array}{l}17,3 \% \\
(21 / 81)\end{array}$ & $\begin{array}{l}30,9 \% \\
(25 / 81)\end{array}$ & $\begin{array}{l}43,2 \% \\
(35 / 81)\end{array}$ \\
\hline $5-6$ & $\begin{array}{c}85 \% \\
(23 / 27)\end{array}$ & $\begin{array}{l}7,4 \% \\
(2 / 27)\end{array}$ & $\begin{array}{c}7,4 \% \\
(2 / 27)^{\star}\end{array}$ & $\begin{array}{l}52,3 \% \\
(23 / 44)\end{array}$ & $\begin{array}{c}25 \% \\
(11 / 44)\end{array}$ & $\begin{array}{c}22,7 \% \\
(10 / 44)^{*}\end{array}$ & & & & $\begin{array}{l}65 \% \\
(46 / 71)\end{array}$ & $\begin{array}{c}18,3 \% \\
(13 / 71)\end{array}$ & $\begin{array}{l}16,9 \% \\
(12 / 71)^{\star}\end{array}$ \\
\hline 9 & & & $\begin{array}{r}11,5 \% \\
(3 / 26)\end{array}$ & & & $\begin{array}{l}40 \% \\
(4 / 10)\end{array}$ & & & & & $\begin{array}{l}5,5 \% \\
(2 / 36)\end{array}$ & $\begin{array}{r}13,9 \% \\
(5 / 36)\end{array}$ \\
\hline 11 & $\begin{array}{l}66,7 \% \\
(14 / 21)\end{array}$ & $\begin{array}{c}23,8 \% \\
(5 / 21\end{array}$ & $\begin{array}{l}9,5 \% \\
(2 / 21)^{*}\end{array}$ & $\begin{array}{l}44,4 \% \\
(16 / 36)\end{array}$ & $\begin{array}{c}19,4 \% \\
(7 / 36)\end{array}$ & $\begin{array}{c}36,1 \% \\
(13 / 36)^{*}\end{array}$ & & & & $\begin{array}{l}52,6 \% \\
(30 / 57)\end{array}$ & $\begin{array}{l}21,1 \% \\
(12 / 57)\end{array}$ & $\begin{array}{l}26,3 \% \\
(15 / 57)^{\star}\end{array}$ \\
\hline 14 & & & $\begin{array}{l}2,9 \% \\
(1 / 34)\end{array}$ & & & $\begin{array}{l}37,5 \% \\
(12 / 32)\end{array}$ & & & & & & $\begin{array}{l}19,7 \% \\
(13 / 66)\end{array}$ \\
\hline Promedio & & & $10,5 \%$ & & & $34,5 \%$ & & & $53,3 \%$ & & & $24 \%$ \\
\hline
\end{tabular}

MF: Mortalidad fetal. MNN: Mortalidad neonatal. SP: Sobrevida perinatal. *: Sobrevida al alta.

$12,9-25 \%(3,4,10)$. En un estudio el $30 \%$ de los niños dados de alta fue con diagnóstico de displasia broncopulmonar (7).

El estudio de Winn y cols en 2000 (3), mostró una sobrevida perinatal global en pacientes con RPM entre las 15 y las 28 semanas de $54 \%$, siendo ésta menor en el grupo estudiado que presentó hipoplasia pulmonar $(95,2 \%)$ que en el que no la tuvo $(48,2 \%)$. Factores de riesgo para desarrollar hipoplasia pulmonar serían una menor EG de RPM, mayor latencia al parto, y menores ILA inicial y promedio (3). Este estudio también muestra que una menor EG de RPM y menor latencia al parto son factores predictores de mortalidad perinatal (3).

$\mathrm{La}$ incidencia de sepsis neonatal reportada fue de $18-63 \%(4,7,10,12,13)$. Algunos estudios muestran una incidencia de hemorragia interventricular grado III o IV de entre 0 a 30\% $(7,12-14)$, retinopatía grado II de $25 \%$ (12) y de enterocolitis necrotizante de $12,5 \%$ (13).

La tasa de malformaciones plásticas y anomalías por compresión varió entre 0-17\% $(4,9,14)$, no relacionando su aparición con el tiempo de latencia en uno de los estudios (4).

Entre los niños dados de alta un trabajo informó que el $9,5 \%$ requería cuidados crónicos y el $47,6 \%$ tenía complicaciones crónicas de prematurez (11). Otro estudio estableció que 8 de 12 niños dados de alta presentaron condiciones médicas crónicas significativas (14). Un estudio que completó un seguimiento a 2 años de 9 niños mostró $50 \%$ de posibilidades de desarrollo neurológico normal (5). Otro no observó alteraciones neurológicas entre los sobrevivientes con seguimiento (10).

\section{DISCUSIÓN}

Los datos obtenidos en esta revisión probablemente subestiman los resultados adversos, debido a que en la mayoría de las poblaciones analizadas existió un número importante de pacientes que optó por la terminación del embarazo y no fueron incluidas en el estudio $(8,5-40,8 \%)(7,8,11,13)$.

Si bien el manejo expectante de la RPM previabilidad está cobrando fuerza en el mundo gracias a los avances en la medicina neonatal, nuestra revisión vuelve a hacer patente la ausencia de guías que estandaricen las intervenciones a realizar, haciendo difícil comparar y extrapolar los resultados maternos y perinatales descritos. Cabe destacar que el rango de sobrevida perinatal obtenido en este estudio va de $2-54 \%$ (promedio $30,1 \%$ ), con una sobrevida al alta del RN de $2-64 \%$ (promedio: $26,8 \%$ ), lo que refuerza la idea que si bien la RPM previabilidad se asocia a un pronóstico reservado, la posibilidad de un resultado favorable no es despreciable al implementar un manejo expectante.

La mayoría de los estudios incluidos mantuvieron hospitalizadas a sus pacientes con RPM del segundo trimestre durante todo el período de latencia, por lo que creemos que ésta conducta es la recomendada. No hay estudios que evalúen 


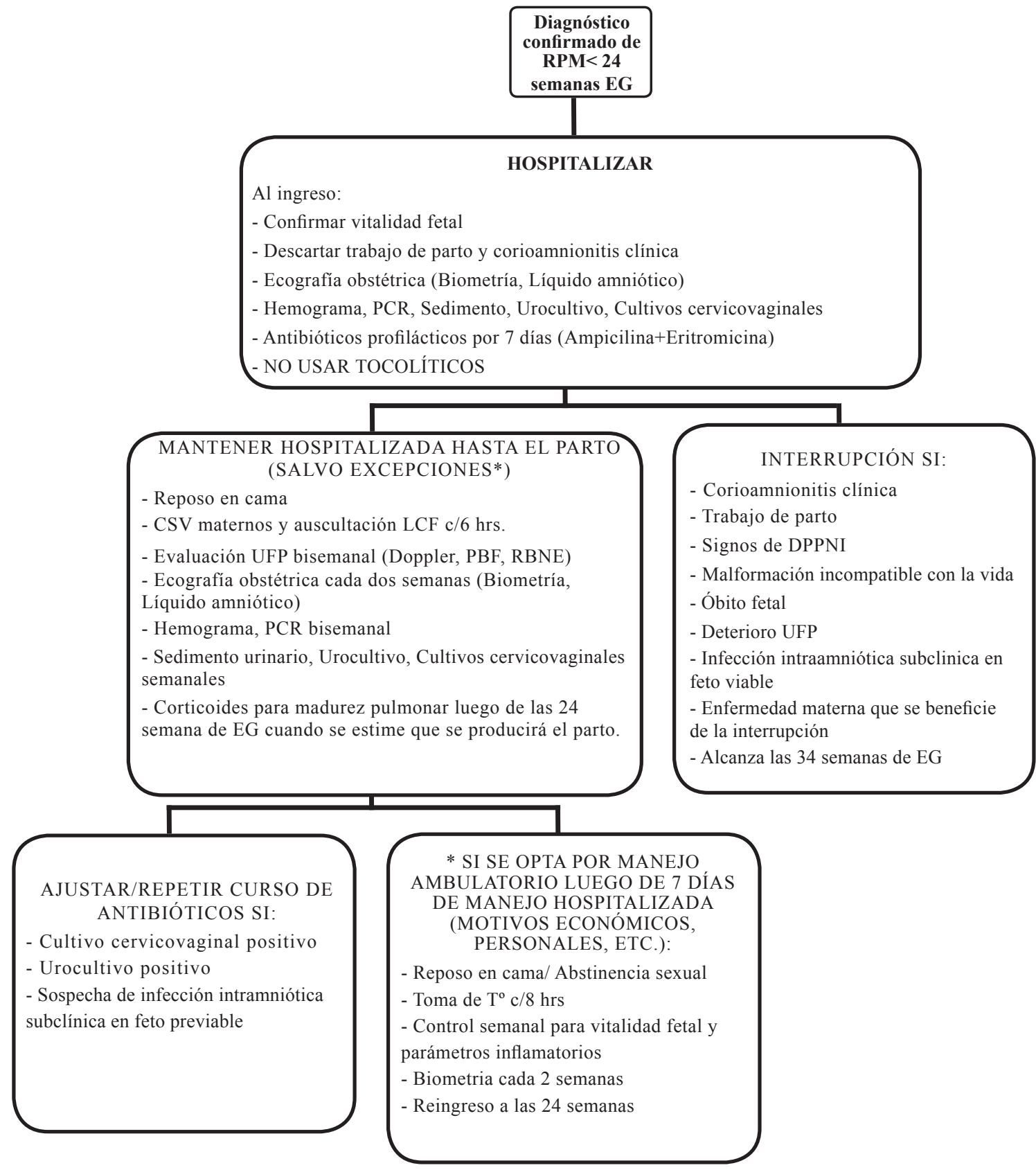

Figura 1. Algoritmo de manejo de la rotura prematura de membranas previabilidad

el manejo hospitalizado versus ambulatorio en la RPM del segundo trimestre, sólo existe evidencia que en RPM de pretérmino no habría beneficio del manejo hospitalizada sobre el ambulatorio de las embarazadas (20), pero no consideramos que esta conducta sea extrapolable a la población de
RPM previable, en la que el riesgo de complicaciones maternas y fetales son mayores.

Recomendamos la toma de cultivos cervicovaginales pues la presencia de gérmenes en este examen se relaciona tanto a la aparición de corioamnionitis clínica como histológica $(4,5)$, y podría 
seleccionar una población de embarazadas que se beneficiaría de tratamiento antibiótico específico, teniendo en cuenta que la corioamnionitis es la principal complicación materna de la RPM del segundo trimestre.

En relación a uso de antibióticos profilácticos su uso está claramente avalado por la literatura una vez alcanzada la viabilidad (21), sin embargo, no hay consenso acerca de su uso en RPM antes de la viabilidad. En nuestra revisión se observa que la indicación de antimicrobianos en este escenario clínico se ha hecho más frecuente que lo observado en revisiones anteriores $(1,2)$, y que su uso podría asociarse a mayor latencia al parto $(5,6,14)$ y que incluso podrían mejorar la sobrevida perinatal $(6,10,12)$. Por este motivo, creemos apropiada la indicación de antibióticos profilácticos al momento de hacer el diagnóstico de RPM del segundo trimestre.

Si bien algunos grupos usaron tocolisis, la evidencia es proclive a contraindicar esta terapia en RPM pues su indicación se asocia a mayor incidencia de corioamnionitis sin mejorar los resultados perinatales (22).

Una vez alcanzada la viabilidad la administración de corticoides es indispensable $(16,17,21)$, cuestión que hoy no se discute en todas las normas internacionales.

\section{CONCLUSIÓN}

Teniendo en cuento los hallazgos descritos, proponemos un algoritmo de manejo de RPM previabilidad (Figura 1). La paciente debe ser informada acerca de la posibilidad de un pronóstico desfavorable de la RPM antes de la viabilidad $(69,9 \%$ de mortalidad perinatal) y debe recibir apoyo emocional de la familia y del equipo médico. Sin embargo, debe ser alentada a un manejo expectante considerando que es posible lograr la sobrevida $(26,8 \%$ de sobrevida al alta) pese al costo emocional y económico del manejo expectante.

\section{REFERENCIAS}

1. Waters TP, Mercer BM. The management of preterm premature rupture of the membranes near the limit of fetal viability. Am J Obstet Gynecol 2009;201:230-40.

2. Dewan H, Morris JM. A systematic review of pregnancy outcome following preterm premature rupture of membranes at a previable gestational age. Aust N Z J Obstet Gynaecol 200;41:389-94.

3. Winn HN, Chen M, Amon E, Leet TL, Shumway JB, Mostello D. Neonatal pulmonary hypoplasia and perinatal mortality in patients with midtrimester rupture of amniotic membranes--a critical analysis. Am J Obstet Gynecol 2000;182:1638-44.

4. Stewart CJ, Tregoning SK, Moller G, Wainwright H.
Preterm prelabour rupture of the membranes before 28 weeks: better than feared outcome of expectant management in Africa. Eur $\mathrm{J}$ ObstetGynecolReprod Biol 2006;126:186-92.

5. Pristauz G, Bauer M, Maurer-Fellbaum U, Rotky-Fast C, Bader AA, Haas J, Lang U. Neonatal outcome and two-year follow-up after expectant management of second trimester rupture of membranes. Int J Gynaecol Obstet 2008;101:264-8.

6. Pristauz G, Bader AA, Schwantzer G, Kutschera J, Lang $U$ Assessment of risk factors for survival of neonates born after second-trimester PPROM. Early Hum Dev 2009;85:177-80.

7. Dinsmoor M, Bachman R, Haney E, Goldstein M, MacKendrick W. Outcomes after expectant management of extremely preterm premature rupture of membranes. Am J Obstet Gynecol 2004;190:183-7.

8. Everest NJ, Jacobs SE, Davis PG, Begg L, Rogerson S. Outcomes following prolonged preterm premature rupture of the membranes. ArchDisChild Fetal Neonatal Ed. 2008 May; 93(3):F207-11.

9. Tews G, Shebl O, Ebner T, Sommergruber M, Jesacher K. Premature rupture of membranes with oligo or anhydramnios before 24 weeks of gestation and the chances of fetal survival. Wien Klin Wochenschr 2004;116:692-4.

10. Xiao ZH, André $P$, Lacaze-Masmonteil T, Audibert $F$, Zupan V, Dehan M. Outcome of premature infants delivered after prolonged premature rupture of membranes before 25 weeks gestation. Eur J Obstet Gynecol Reprod Med 2000;90:67-71.

11. Falk S, Campbell L, Lee-Parritz A, Cohen A, Ecker J, Wilkins-Haug L, Lieberman E. Expectant management in spontaneous preterm premature rupture of membranes between 14 and 24 weeks' gestation. J Perinatol 2004;24:611-6.

12. Grisaru-Granovsky S, Eitan R, Kaplan M, Samueloff A. Expectant management of midtrimester premature rupture of membranes: a plea for limits. J Perinatol 2003;23:235-9.

13. Muris C, Girard B, Creveuil C, Durin L, Herlicoviez $M$, Dreyfus M. Management of premature rupture of membranes before 25 weeks. Eur J Obstet Gynecol Reprod Biol 2007;131:163-8.

14. Verma U, Goharkhay N, Beydoun S. Conservative management of preterm premature rupture of membranes between 18 and 23 weeks of gestation--maternal and neonatal outcome. Eur J Obstet Gynecol Reprod Biol 2006;128:119-24.

15. Scholz HS, Arikan MG, Benedicic C, Petru E, Haas $\mathrm{J}$, Weiss PA. Outcome of pregnancies after non-amniocentesis-induced premature rupture of membranes at 14 to 23 weeks' gestation. Wien Klin Wochenschr 2002; $114: 50-3$.

16. Roberts D, Dalziel S. Antenatal corticosteroids for accelerating fetal lung maturation for women at risk of preterm birth. Cochrane Database Syst Rev 2006;3:CD004454.

17. Harding JE, Pang JM, Knight DB, Liggins GC. Do antenatal corticosteroids help in the setting of preterm rupture of membranes? Am J ObstetGynecol 2001;184:131-9.

18. Hadi HA, Hodson CA, Stickland D. Premature rupture 
of membranes between 20 and 25 weeks' gestation: role of amniotic fluid volume in perinatal outcome. Am J Obstet Gynecol 1994;170:1139-44.

19. Burrows LJ, Meyn LA, Weber AM. Maternal morbidity associated with vaginal versus cesarean delivery. Obstet Gynecol 2004;103:907-12.

20. Abou El Senoun G, Dowswell T, Mousa HA. Planned home versus hospital care for preterm prelabour rupture of the membranes (PPROM) prior to
37 weeks' gestation. Cochrane Database Syst Rev 2010;4:CD008053.

21. Kenyon S, Boulvain M, Neilson JP. Antibiotics for preterm rupture of membranes. Cochrane Database Syst Rev 2010;8:CD001058.

22. Mackeen AD, Seibel-Seamon J, Grimes-Dennis J, Baxter JK, Berghella V. Tocolytics for preterm premature rupture of membranes. Cochrane Database of Syst Rev 2011;10:CD007062. 